\title{
Labyrinthe
}

32 | 2009 (1)

Le petit théâtre intellectuel

\section{Le Débatteur}

\section{Renaud Pasquier}

\section{OpenEdition}

\section{Journals}

Édition électronique

URL : http://journals.openedition.org/labyrinthe/3983

DOI : $10.4000 /$ labyrinthe.3983

ISSN : 1950-6031

Éditeur

Hermann

Édition imprimée

Date de publication : 19 juin 2009

Pagination : 29-32

ISBN : 978-2-7056-6885-3

\section{Référence électronique}

Renaud Pasquier, « Le Débatteur », Labyrinthe [En ligne], 32 | 2009 (1), mis en ligne le 01 février 2011. consulté le 01 mai 2019. URL : http://journals.openedition.org/labyrinthe/3983 ; DOI : 10.4000/ labyrinthe.3983 


\section{Le petit théâtre intellectuel}

\section{LE DÉBATTEUR}

Au commencement il y avait la conférence. Elle était solennelle, sérieuse, mais aussi mondaine. Le public était choisi, silencieux, déférent. Du haut de sa chaire, le Conférencier lui dispensait son savoir d'un verbe noble et sentencieux; il avait de fines moustaches, portait une redingote, roulait les « $\mathrm{r}$ » et scandait des périodes savamment composées. Son discours était sans trouble et sa voix apaisante. La conférence osait parfois se faire causerie, délaissant la gravité ecclésiastique du prêche qui l'imprégnait d'ordinaire. Tout cela n'est plus. La conférence demeure, mais elle ne concerne plus le savoir, bien plus le pouvoir: elle est « internationale » et toujours « au sommet», loin des simples mortels. Sous d'autres formes elle ne subsiste guère, résidu couleur sépia d'une époque lointaine, une Troisième République valéryenne perdue dans les brumes du temps. La vétusté sied du reste au Conférencier, homme de haute culture et amant du passé: il se mue souvent en Pythie, quand il n'est pas relégué au divertissement touristique, cicerone nostalgique et érudit, mais aussi vestige lui-même, voire carrément Momie. Rideau.

"Le présent est à moi! ", parade le Débatteur, surgi sur la scène. De tous ceux qui peuplent notre " petit théâtre ", il est le prétendant le plus acharné au trône, à la couronne et au sceptre: si ce n'est le titre, au moins la majuscule du mot «Intellectuel ». Il a - toujours - des arguments, il brandit son arbre généalogique, me le colle sous le nez en clamant: «Zola! Voltaire! Démosthène! Socrate! Car Socrate - son ton se fait grave - a fait descendre la philosophie du ciel sur la terre, parmi les hommes ». Et puis Socrate n'écrit pas, il parle. Le Débatteur en rêve parfois; car le livre ne lui suffit pas, souvent il s'en méfie, il préfère l'article, me confie-t-il. Inquiet de l'ici et du maintenant, il veut s'adresser à tous, du moins au plus grand nombre, et il y parvient avec un réel talent; il a la hantise de la Tour d'ivoire et ses avatars, comme cette bibliothèque confinée où le Conférencier tenait salon. "Mes phrases sont de combat, elles sont nées dans les tribunaux échauffés, les assemblées tumultueuses, les rues en lutte! Me faire taire, c'est bâillonner la Démocratie! " m'assène le Débatteur en tapant du poing (le faire taire? Qui y parviendrait?). Il est vrai 
qu'avec lui, la rigide verticalité conférencière fait place à une circulation horizontale du discours qui bouscule les hiérarchies. Le Conférencier était un soliste (parfois) virtuose, la voix du Débatteur n'existe qu'au sein de la polyphonie orchestrale, plus exactement dans le contrepoint. Ami du Polémiste - ils se querellent de bon cœur, quotidiennement - il n'aime pas l'harmonie et se défie de l'accord. "Penser c'est dire non » est sa citation favorite (de qui? Il ne sait plus), et il y obéit avec un indéniable génie: dans les opinions les mieux reçues et les sujets les plus triviaux, dans les discours savants les plus ésotériques censément réservés aux initiés, il décèle la polémique en puissance, s'emploie à semer le doute, à mettre au jour la faille où la discorde peut s'engouffrer. Alors il ouvre le feu; l'adversaire réplique; les camps s'organisent: la controverse est lancée. Soufflant en rafales, elle s'introduit dans les foyers, anime les discussions, envahit les esprits. Tout le monde est concerné, chacun est sommé de se déclarer. Le Débatteur jubile, dans son élément. Benoîte est sa tête de turc favorite: il méprise ses discours iréniques et lénifiants, résidus d'un autre âge (celui du Conférencier). «Je suis un Moderne, $m o i$ », me rappelle-t-il en se frappant la poitrine. De fait son nom vient de l'anglais (debater). "Moderne, je te dis! ». Il est né en effet, en tant que professionnel patenté, il y a trois ou quatre décennies.

Beaucoup l'appellent Bavard ou Baratineur. L'Expert-ils se détestent - le dit incompétent. Il hausse les épaules: "Des jaloux, des envieux, voilà tout. Ils sont moins connus que moi ». Je ne lui donne pas tort. Car il est partout, dans la presse, en tribune, sur la scène, en studio, à l'écran. Un peu trop, peut-être?, lui dis-je timidement. Il balaie l'objection: "Il faut y aller, occuper l'terrain, c'est là qu'ça se passe ». Il distille formules, anecdotes, images, passe d'un registre à l'autre sans dommage. Ses certitudes et son aisance irritent mais séduisent et charment. Pas de conférence pour lui, mais des communications, des interventions, des tables rondes, et bien sûr des débats: parole collective, toujours, mais surtout conflictuelle - «non : vivante! », me corrige-t-il. Le Spécialiste, ironique, lui demande quand il trouve le temps de travailler. La réponse fuse: "Quand j'écris, moi, j'ai plus de vingt lecteurs. Je ne suis pas coincé, moi, dans mon petit secteur, mon petit savoir. Moi, je suis in-disci-pli-né ». Pas question de s'enfermer dans son domaine, il prend la parole en toute occasion et à tout propos (en Italie, on l'appelle parfois Tuttologo, le «toutologue »). L'air songeur, il m'avoue qu'au fond, il 


\section{Le Débatteur}

se sent plus proche des journalistes. Mais ce n'est pas le moment de gamberger, dans une heure, " $y$ a débat », il faut se préparer.

" Repérer les points faibles, frapper là où ça fait mal », se répète-t-il, boxeur avant son combat. "Pas le lâcher, pas lui laisser une seconde". Le Débatteur parle vite, c'est une mitraillette verbale. Il est entraîné, il a du souffle, il dure. Car tout est là : dire plus, plus fort, plus vite, plus longtemps. Athlète de la parole, le Débatteur se débat, il aime le corps à corps, il veut la victoire. N'hésite pas à tricher. Interrompt l'adversaire. Frappe en dessous de la ceinture: vanne méchante, ricanement, il se grime en Comique. Quand il est dans l'arène, les rires du public le galvanisent. Nous sommes au Talk Show, la parole se donne en spectacle.

«C'est un bon client» disent de lui, justement, les promoteurs de spectacle. Prêtons l'oreille au jargon, qui dit vrai, et complétons-le: le « bon client » est aussi un marchand de phrases, qui vit de son commerce. Dans les deux cas, offre et demande, le Débatteur fait tourner la machine: trublion, certes, mais juste ce qu'il faut. Il remplit docilement son rôle dans un dispositif bien réglé: les structures binaires sont préétablies, pour/contre, gauche/droite, jeunes/vieux, Nouveaux Réacs/Rebelles Éternels etc., il suffit de s'y installer, il est permis de changer de place. Le Débatteur, même hargneux, ne prend pas de risques: il reste dans le périmètre à lui imparti. Il le dit et le croit suffisant pour pouvoir «faire passer des trucs, réveiller les consciences, faire bouger les choses, tu vois ».

Cependant, dans le feu de l'action, il ne pense pas. Il ferraille. Son métier c'est attaquer, répondre, répondre aux réponses. Je l'écoute et me perds parfois dans les méandres de son raisonnement, tant il veut retourner les doxas, finissant à la longue par tenir les discours de ses ennemis d'hier (le Renégat lui a appris ses tours). À force de simplification, de vulgarisation, ses propos, vieux outils usés, ont perdu toute consistance, émoussés et insipides. Je n'arrive plus à suivre le ping-pong verbal auquel il joue avec son partenaire/adversaire, je ne sais plus de quoi ils parlent avec tant d'ardeur, je ne les distingue plus, silhouettes interchangeables, j'ai le tournis. Peu à peu leurs voix se fondent en une seule mélopée aux rythmes ondulants, étonnamment proche des mornes litanies du Conférencier. Je sombre dans un sommeil profond.

R. P. 


\section{Bibliographie, références, lectures}

I. Paul Valéry, notamment dans Regards sur le monde actuel (Paris, Folio essais, 2002), représente sans doute la quintessence, et la version la plus haute du Conférencier.

II. Toujours sur le Conférencier, on pourra s'amuser à lire un des Tropismes de Nathalie Sarraute consacrée à un professeur au Collège de France, cf. Tropismes, XII, Paris, Minuit, 1957, pp. 77-78.

III. Jacques Rancière expose dans La Mésentente (Paris, Galilée, 1995) comment toute politique réelle se manifeste dans une énonciation polémique (apparent triomphe du débatteur) mais aussi comment celle-ci remet en question le socle même du débat, par intervention d'un Tiers non admis dans la discussion (contre le Débatteur, et, en l'occurrence contre Habermas).

IV. La lecture de quelques éditoriaux de Labyrinthe où nous chantons l'indiscipline ne me paraît pas ici sans pertinence.

V. Gilles Deleuze a des phrases définitives (dont l'une figure dans ce texte, empruntée) dans un entretien "À propos des nouveaux philosophes et d'un problème plus général », disponible en ligne à l'adresse http://multitudes. samizdat.net/A-propos-des-nouveaux-philosophes [consulté le 15 février 2009].

VI. Le personnage de Calliclès dans le Gorgias de Platon peut être considéré comme un archétype du Débatteur.

VII. Dans la deuxième partie («Un grand homme de province à Paris ») d'Illusions perdues de Balzac, plusieurs critiques littéraires donnent à Lucien Chardon une leçon sur le métier, qui suppose d'envisager chaque idée selon «son envers et son endroit». Quelques mots de cette page figurent dans le texte. 